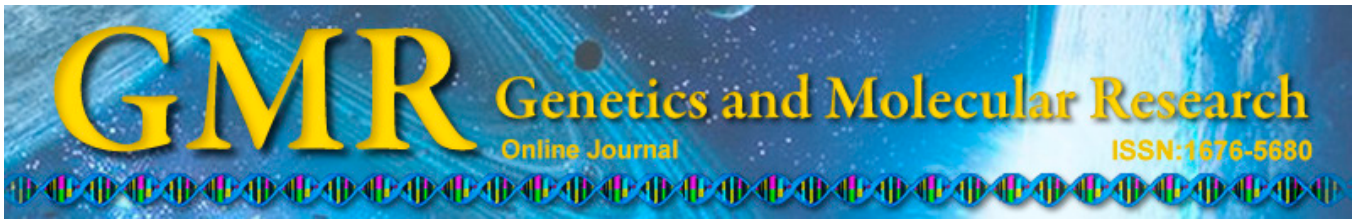

\title{
Inactivation of Rab23 inhibits the invasion and motility of pancreatic duct adenocarcinoma
}

\author{
Z.Z. Cai ${ }^{1}$, L.B. Xu ${ }^{2}$, J.L. Cai ${ }^{2,3}$, J.S. Wang ${ }^{2}$, B. Zhou ${ }^{2}$ and H. $\mathrm{Hu}^{3}$ \\ ${ }^{1}$ Department of Gastroenterology, \\ The Second Affiliated Hospital of Wenzhou Medical University, Wenzhou, China \\ ${ }^{2}$ Department of General Surgery, \\ The Second Affiliated Hospital of Wenzhou Medical University, Wenzhou, China \\ ${ }^{3}$ Department of General Surgery, \\ East Hospital Tongji University School of Medicine, Shanghai, China \\ Corresponding author: J.L. Cai \\ E-mail: jinglicai@hotmail.com
}

Genet. Mol. Res. 14 (1): 2707-2715 (2015)

Received June 5, 2014

Accepted October 20, 2014

Published March 30, 2015

DOI http://dx.doi.org/10.4238/2015.March.30.31

\begin{abstract}
Pancreatic ductal adenocarcinoma (PDAC) is characterized by a poor prognosis and high mortality rate. In this study, we investigated the expression of Rab23 in non-tumor pancreatic tissues and PDACs via immunohistochemistry. Rab23 was found in 39 of 58 $(67.2 \%)$ and in 11 of $30(36.7 \%)$ of the PDAC and non-tumor pancreatic tissue samples $(P=0.0073)$, respectively. There were significant correlations between Rab23 expression and unfavorable variables, including cancer differentiation level $(\mathrm{P}=0.0089)$, lymph nodal $(\mathrm{P}=$ $0.0099)$, and distant metastases $(P=0.0173)$. Inactivation with small interfering RNA against Rab23 in the human pancreatic cancer cell line Panc-1 inhibited the migration and invasive potential of the cells. Our data provide new insight into the essential role of Rab23 in PDAC invasion and metastasis and suggest that Rab23 expression is a useful indicator of metastatic potential; hence, it may be a new therapeutic target for this common malignancy.
\end{abstract}

Key words: Migration; Pancreatic duct adenocarcinoma; Rab23; siRNA 


\section{INTRODUCTION}

Pancreatic cancer is one of most lethal malignancies, has one of the poorest prognoses, and is the 4th leading cause of tumor-associated mortality worldwide (Michaud, 2004; Chakraborty et al., 2011). According to the US Centers for Disease Control and Prevention, although pancreatic cancer accounts for only $2 \%$ of all new cancers each year, its incidence is nearly equal to its mortality rate, and the 5-year survival rate of $4-5 \%$ is the lowest among all cancers (Jemal et al., 2006; Koorstra et al., 2008). Pancreatic ductal adenocarcinoma (PDAC) comprises over $90 \%$ of cases of pancreatic cancers and is characterized by aggressive local invasion, extensive regional lymph node metastasis, and distant metastasis during the early stages of carcinogenesis (Silverman et al., 1994; Jemal et al., 2006). At the time of diagnosis, most patients already exhibit locally advanced or metastatic disease, which limits the therapeutic value of surgical resection of the primary tumor (Roy et al., 2014). Moreover, PDACs exhibit poor responses to chemo- and radiotherapy and a tendency for recurrence because of their aggressive nature (Dey et al., 2012). Therefore, an improved understanding of the molecular mechanisms of PDAC carcinogenesis is urgently needed and novel molecular targets must be identified to improve the current management of PDAC.

Rab GTPases play critical roles in regulating intercellular vesicle trafficking, signal transduction, and receptor recycling, and these processes in turn regulate normal membrane polarity (Cheng et al., 2004; Caswell et al., 2007). Rab23 is a novel Ras-related small GTPase, the gene which is located in the pericentromeric region of chromosome 6 between the microsatellite markers D6S257 and D6S1659 within the critical region of RP25. The gene is composed of 7 exons spanning a 34-kb domain of genomic DNA (Marcos et al., 2003). Mammalian Rab23 localizes to the plasma membrane, endosomes, and cytosol and functions as an essential regulator of nervous and skeletal system differentiation via genetic interactions with Sonic hedgehog (Yang et al., 2008; Chi et al., 2012). Recent studies have confirmed Rab23 is amplified in primary tumors, such as hepatocellular carcinoma and diffuse-type gastric cancer, and have provided functional evidence that Rab23 regulates the cell proliferation and invasion of tumors (Liu et al., 2007; Hou et al., 2008). However, no reports regarding the expression and function of Rab23 in PDAC are available. In the present study, we found that increases in Rab23 expression contributed to the invasion and metastasis of PDACs.

\section{MATERIAL AND METHODS}

\section{Patients and tissue samples}

Human PDAC samples $(\mathrm{N}=58)$ were randomly collected from surgery specimens at the Department of Minimally Invasive Surgery, the Second Affiliated Hospital of Wenzhou Medical College, Wenzhou, China, between 2012 and 2013. None of the patients received radiotherapy or chemotherapy prior to surgery. Additionally, non-tumor pancreatic specimens were obtained from 30 patients with chronic pancreatitis who had undergone surgery. All tissue samples were fixed in 10\% formalin and embedded in paraffin for immunohistochemical analyses. The diagnoses were verified by pathologists in our hospital. All samples were obtained with informed consent, and the protocol for this study was approved by the Ethics Committee of the hospital. 


\section{Immunohistochemistry}

Paraffin-embedded tissue sections (6- $\mu \mathrm{m}$ thick) were dewaxed, rehydrated, and immersed in methanol containing $0.3 \%$ hydrogen peroxide for 30 min to block endogenous peroxidase activity. Subsequently, the sections were heated in a microwave oven filled with Tris-buffered citric acid, $\mathrm{pH}$ 6, for 10 min for antigen retrieval. Primary Rab23 polyclonal antibodies (BD Biosciences, Franklin Lakes, NJ, USA) were diluted to 1:200 and incubated with the sections for $2 \mathrm{~h}$ at room temperature. Detection was performed using Elivision TMPlus Polymer HRP (mouse/rabbit) immunohistochemistry kits (Maixin-Bio, Fuzhou, China). The visualization signal was developed using diaminobenzidine.

\section{Cell culture}

Cells from the Panc-1 human PDAC cell line were obtained from the American Type Culture Collection and were grown in DMEM high-glucose (Gibco, Grand Island, NY, USA) supplemented with $100 \mathrm{IU} / \mathrm{mL}$ penicillin, $100 \mu \mathrm{g} / \mathrm{mL}$ streptomycin, and $10 \%$ fetal bovine serum (Hyclone, Logan, UT, USA) in a $37^{\circ} \mathrm{C}$ and $5 \% \mathrm{CO}_{2}$ cell culture incubator.

\section{siRNA transfection}

The human Rab23 targeting siRNA and non-targeting control siRNA were purchased from Dharmacon (Lafayette, CO, USA). Dharma FCET1 reagent (Dharmacon) was used to transfect the siRNA according to the manufacturer instructions as previously described (Liu et al., 2012). Assays were performed $48 \mathrm{~h}$ after transfection.

\section{RNA extraction and reverse transcription-polymerase chain reaction (RT-PCR)}

Total cellular RNA was extracted from cells using TRIzol reagent (Sigma, St. Louis, MO, USA) according to manufacturer instructions. Reverse transcription with oligo (dT) priming (Promega, Madison, WI, USA) was used to generate cDNAs from $1 \mu \mathrm{g}$ total RNA. Expression was quantitatively analyzed using a standard SYBR green PCR kit (Promega), and PCR-specific amplification was conducted in an Eppendorf real-time PCR machine (Hamburg, Germany). Gene expression was calculated using the $2^{-(\Delta \Delta \mathrm{Ct})}$ method. The primer sequences were as follows: Rab23 sense, 5'-AGCGA GACTCCGTCTTCAAA-3'; Rab23 antisense, 5'-CACCCCTAAGGTACG CATGT-3'; glyceraldehyde 3-phosphate dehydrogenase sense, 5'-GCACCGTCAAGG CTGAGA AC-3'; and glyceraldehyde 3-phosphate dehydrogenase antisense, 5'-TGGTGAAGA CGCCAG TGGA-3'.

\section{Western blot assays}

The cells were washed with cold phosphate-buffered saline and lysed on ice with RIPA lysis buffer containing $150 \mathrm{mM} \mathrm{NaCl}, 1 \% \mathrm{NP} 40,0.5 \%$ sodium deoxycholate, $0.1 \%$ sodium dodecyl sulfate, $50 \mathrm{mM}$ Tris, $\mathrm{pH}$ 7.9, $10 \mathrm{mM} \mathrm{NaF}$, phenylmethylsulfonyl fluoride, and protease inhibitor cocktail (Sigma, St. Louis, MO, USA). The protein concentrations were determined using a Bio-Rad protein assay kit (Hercules, CA, USA). The cell extracts (50 $\mu \mathrm{g} /$ lane) were separated by sodium dodecyl sulfate-polyacrylamide gel electrophoresis and trans- 
ferred onto polyvinylidene fluoride membranes. The blots were probed with human Rab23 antibody (final dilution 1:1000, Sigma). Glyceraldehyde 3-phosphate dehydrogenase antibody (final dilution 1:1000, Sigma) was used as a control. The bands were detected using the enhanced chemiluminiscence kit (GE Healthcare, Little Chalfont, UK) and visualized using the ChemiDoc XRS system (Bio-Rad).

\section{Transwell migration assay}

Transwell migration assays were performed using 6.5 - $\mathrm{mm}$ diameter cell culture inserts $(8-\mu \mathrm{m}$ pore size; Costar, Corning, NY, USA) on 24-well culture plates. The cells were trypsinized and suspended in serum-free Dulbecco's modified eagle medium high-glucose. The suspended cells $\left(2 \times 10^{4}\right.$ per well) in serum-free medium were added to the upper chamber of the transwell insert, and complete medium was added to the lower chamber of the transwell insert. Following $24 \mathrm{~h}$ of incubation, the inserts were washed with phosphate-buffered saline. Adherent cells on the inner side of the membrane of the upper chamber were removed from the upper surface using cotton tips. Invading cells that had adhered to the bottom of the membrane were fixed in cold methanol for $10 \mathrm{~min}$, stained with crystal violet for $15 \mathrm{~min}$, and counted under a microscope.

\section{Scratch-wound assay}

Panc-1 cells were seeded onto 6-well plates $\left(5 \times 10^{5}\right.$ per well). After the cells grew to $90 \%$ confluence, the cell monolayers were wounded manually by scratching with a 200 $\mu \mathrm{L}$ pipette tip. The exfoliated cells were washed off using phosphate-buffered saline, and the culture was continued in fresh medium. Wound closure was monitored by microscopy at $24 \mathrm{~h}$ using a Nikon inverted microscope (Nikon, Tokyo, Japan). Migration activity was calculated as the percentage of wound closure using the initial scratch width as $100 \%$. Experiments were performed in triplicate for each treatment group.

\section{Statistical analysis}

The SPSS 17.0 software (SPSS, Chicago, IL, USA) was used for all statistical analysis. The values are reported as means \pm standard deviation of at least 3 repeated individual experiments for each group. Significant differences were determined using the Student $t$-tests to compare 2 groups. Significance was accepted at the level of $\mathrm{P}<0.05$.

\section{RESULTS}

\section{Expression of Rab23 in non-tumor pancreatic tissues and PDACs by immunohistochemistry}

To investigate the characteristics of Rab23 protein expression in non-tumor pancreatic tissues and PDACs, 30 chronic pancreatitis tissue samples and 58 PDAC samples were immunohistochemically analyzed. The immunostaining data are summarized in Table 1 . We found that Rab23 was present in 39 of 58 (67.2\%) PDAC samples and 11 of 30 (36.7\%) nontumor pancreatic tissues $(\mathrm{P}=0.0073)$ (Figure 1A and $\mathrm{B})$. Furthermore, Rab23 expression was 
significantly correlated with unfavorable variables that included cancer differentiation level ( $\mathrm{P}$ $=0.0089)$ and lymph node $(\mathrm{P}=0.0099)$ and distant metastases $(\mathrm{P}=0.0173)$. However, there were no significant differences between groups when separated according to any other clinicopathologic feature, such as gender, age, tumor size, or TNM stage.

Table 1. Correlations between Rab23 expression in pancreatic duct cancer and clinical characteristics.

\begin{tabular}{|c|c|c|c|c|}
\hline \multirow[t]{2}{*}{ Group } & \multirow[t]{2}{*}{$\mathrm{N}$} & \multicolumn{2}{|c|}{ Rab23 expression } & \multirow[t]{2}{*}{ P value } \\
\hline & & Positive & Negative & \\
\hline Non-tumor tissue & 30 & 11 & 19 & 0.0073 \\
\hline Cancer tissue & 58 & 39 & 19 & \\
\hline \multicolumn{5}{|l|}{ Gender } \\
\hline Male & 32 & 18 & 14 & 0.7919 \\
\hline Female & 26 & 13 & 13 & \\
\hline \multicolumn{5}{|l|}{ Age } \\
\hline$>60$ & 36 & 17 & 19 & 0.7871 \\
\hline$\leq 60$ & 22 & 12 & 10 & \\
\hline \multicolumn{5}{|l|}{ Size of carcinoma $(\mathrm{cm})$} \\
\hline$>3$ & 26 & 16 & 10 & 0.3004 \\
\hline$\leq 3$ & 32 & 15 & 17 & \\
\hline \multicolumn{5}{|l|}{ Degree of differentiation } \\
\hline Well and moderately differentiation & 31 & 12 & 19 & 0.0089 \\
\hline Poorly differentiation & 27 & 20 & 7 & \\
\hline \multicolumn{5}{|l|}{ TNM stage } \\
\hline I-II & 23 & 9 & 4 & 0.2829 \\
\hline III & 35 & 20 & 15 & \\
\hline \multicolumn{5}{|l|}{ Lymph node metastasis } \\
\hline Negative & 27 & 8 & 19 & 0.0099 \\
\hline Positive & 31 & 20 & 11 & \\
\hline \multicolumn{5}{|l|}{ Distant metastasis } \\
\hline Negative & 29 & 9 & 20 & 0.0173 \\
\hline Positive & 29 & 19 & 10 & \\
\hline
\end{tabular}

Based on the American Joint Committee on Cancer/International Union Against Cancer staging manual (2009). Differences between the variables were assessed with the Fisher exact tests.

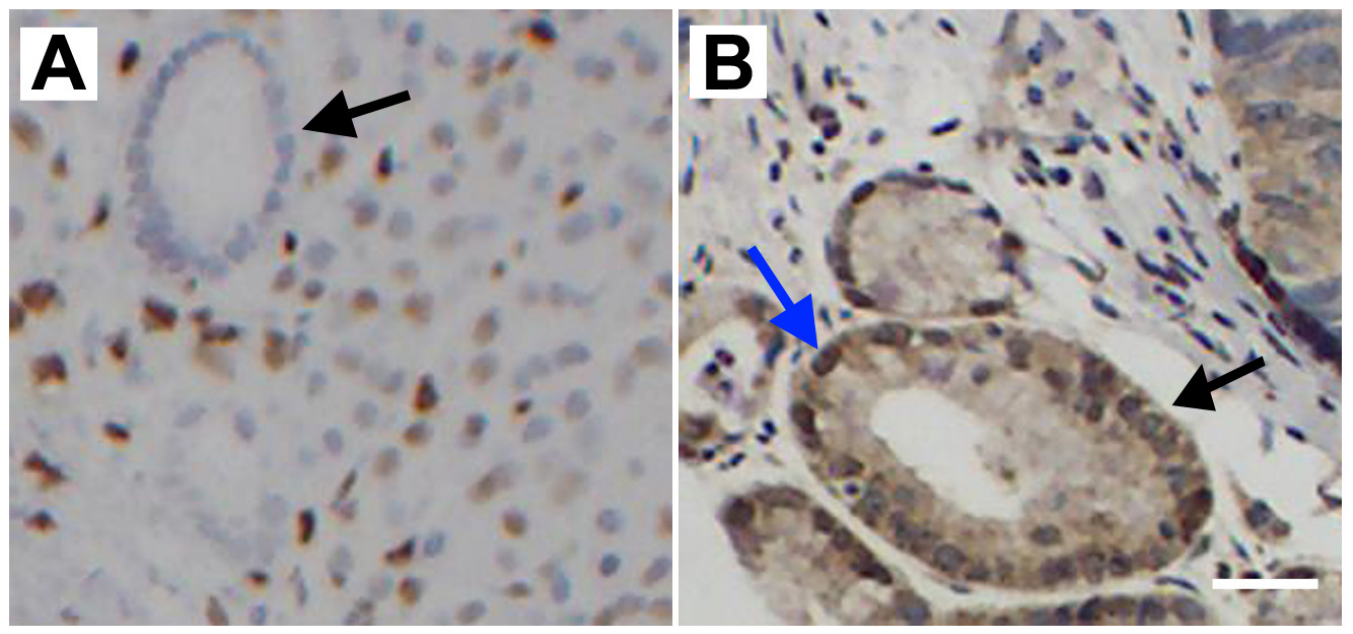

Figure 1. Expression of Rab23 in non-tumor tissues and PDACs revealed by immunohistochemistry. A. Non-tumor pancreatic tissue. B. PDAC tissue (scale bar $=50 \mu \mathrm{m}$ ). The black and blue arrows indicate the pancreatic duct structure and Rab23-positive cells, respectively. 


\section{siRNA decreased Rab23 expression in Panc-1 cells}

The siRNA targeting Rab23 that was ubiquitously produced was used to block Rab23 expression in Panc-1 cells. To investigate the knockdown efficiency of Rab23 siRNA in Panc-1 cells, the mRNA levels of Panc-1 cells were evaluated by RT-PCR after siRNA transfection. The results revealed that Rab23 siRNA reduced the Rab23 mRNA level by 55.4\% in Panc-1 cells (Figure 2A). Western blot analysis revealed that the Rab23 protein level was dramatically decreased by $74.5 \%$ compared to the control groups following Rab25 siRNA transfection (Figure 2B).

\section{A}

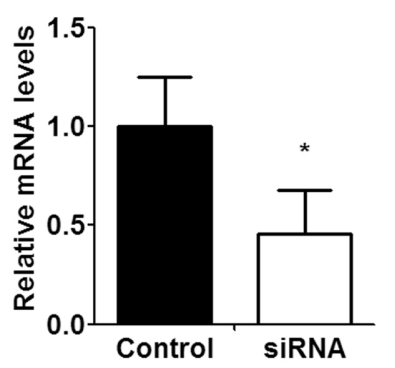

B

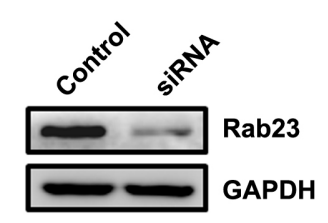

Figure 2. Knockdown of Rab23 expression in Panc-1 cells. Panc-1 cells were transfected with control or Rab23 siRNA for 48 h. A. Rab23 mRNA expression in the Panc-1 cells was measured by real-time PCR. Data are reported as means \pm $\mathrm{SD}(\mathrm{N}=3) . * \mathrm{P}<0.05$ vs the control group. B. Western blot analysis of Rab23 expression. GAPDH was analyzed as the loading control. Experiments were performed in triplicate. Representative photographs are shown.

\section{Knockdown of Rab23 inhibited the invasion and migration of Panc-1 cells in vitro}

Next, we examined the effects of Rab23 downregulation on the invasion and migration properties of Panc-1 cells. The transwell assay was used to detect cell invasion. Compared to the control group, transfection with Rab23 siRNA resulted in a $56.1 \%$ reduction in the migrated cells in the chamber of the trans-well plate (Figure 3A and B). Furthermore, we performed cell migration wound-healing assays. Treatment of Panc-1 cells with Rab23 siRNA resulted in $45.7 \%$ inhibition of cell migration (Figure 4A and B). These results support the functional invasion-mediating role of Rab23 in PDACs.

A

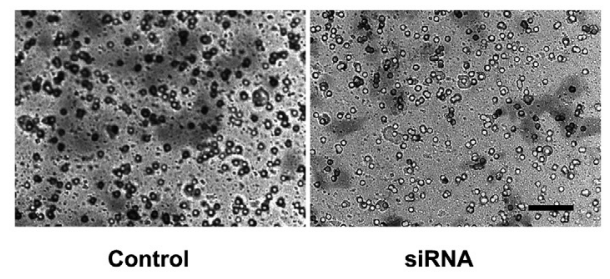

B

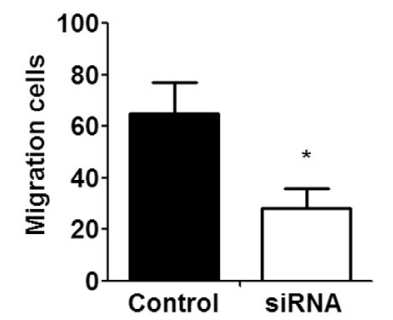

Figure 3. Cell invasion abilities of the Panc-1 cells as measured using transwell assays. Panc-1 cells were transfected with control or Rab23 siRNA for $48 \mathrm{~h}$. A. Representative images of the transmembrane cells after $24 \mathrm{~h}$ of incubation (scale bar $=50 \mu \mathrm{m}$ ). B. Transmembrane cells were counted in 5 high-power fields of each chamber. The results are reported as means $\pm \mathrm{SD}$. $* \mathrm{P}<0.05$ vs control group, $\mathrm{N}=3$. 
A

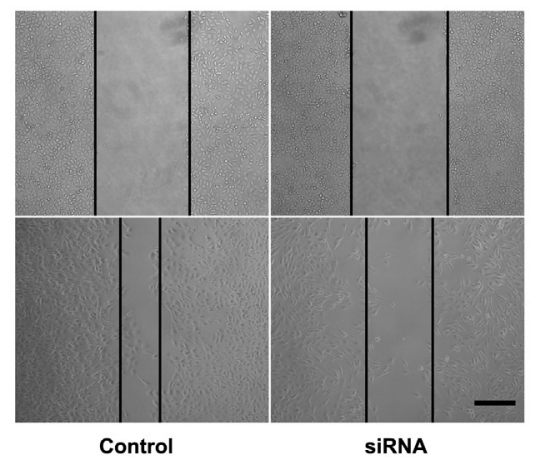

B

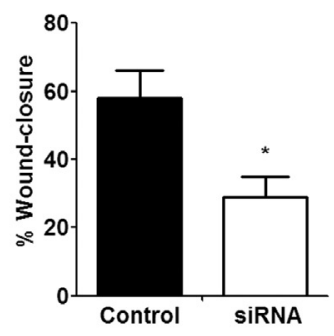

Figure 4. Cell migration ability of Panc-1 cells as assessed using a scratch-wound assay. Panc-1 cells were transfected with control or Rab23 siRNA for $48 \mathrm{~h}$. A. Representative images were taken at 0 and $24 \mathrm{~h}$ to assess the cell migration into the open space (scale $b a r=100 \mu \mathrm{m}$ ). B. The percentage of the distance migrated was determined by measuring these distances in 5 high-power fields. The results are reported as means $\pm \mathrm{SD}$. ${ }^{*} \mathrm{P}<0.05 v s$ the control group, $\mathrm{N}=3$.

\section{DISCUSSION}

The prevalence and mortality rate of PDAC is expected to rise over the next decade. Although advanced molecular therapeutic agents such as erlotinib (Moore et al., 2007) have emerged in the past decade, PDAC remains a lethal disease with a limited number of available therapeutic options. PDAC carcinogenesis is complex and involves the sequential acquisition of a variety of genetic alterations (Jones et al., 2008). PDAC is characterized by a multistage process associated with the pathway of a few factors that activate the cell cycle and promote mitosis or inhibit apoptosis, including mitogen-activated protein kinase, nuclear factor- $\mathrm{\kappa B}$, SMAD, and hedgehog signaling pathways (Abbas, 2013).

Rab23 plays essential roles by facilitating vesicular transport and controlling endocytic progression to the lysosomes (Guo et al., 2006; Smith et al., 2007). Unlike other Rab proteins, the Rab23 protein is present in the nucleus and cytoplasm (Huang et al., 2011), suggesting that Rab23 has other specific functions in addition to membrane trafficking. Rab23 is an essential negative regulator of the hedgehog pathway (Eggenschwiler et al., 2001) and acts in a hedgehog-independent manner by regulating the expression of a specific downstream component of this signaling pathway (Eggenschwiler et al., 2006). Dysregulation of the hedgehog pathway has been implicated in the genesis of several types of cancer (Beachy et al., 2004). Rab23 has recently been implicated in carcinogenesis. High Rab23 expression levels have been reported in hepatocellular and gastric carcinomas (Liu et al., 2007), and siRNA silencing of Rab23 significantly reduces cellular invasion and migration, indicating that Rab23 is an invasion-mediating gene in gastric cancer (Hou et al., 2008).

Our findings demonstrated that Rab23 is overexpressed in PDAC tissue and thus validated the role of the Rab23 gene as a mediator of metastasis in PDAC patients. Functionally, siRNA-mediated knockdown of Rab23 inhibited the invasive capacity of Panc-1 cells, suggesting that Rab23 plays a role in PDAC cell invasion. Our data strongly support the results of previous studies suggesting that Rab23 plays roles in tumorigenesis and cancer cell invasion. However, the mechanisms by which Rab23 contributes to pancreatic tumorigenesis remain 
poorly understood. Recent studies have established the functional role of hedgehog signaling in metastatic processes in PDAC, which is mediated primarily through signaling pathway activation (Morifuji et al., 2014). Furthermore, elevated levels of hedgehog pathway components have been observed in PDAC precursor lesions, and these levels increase further as the lesions progress to more advanced stages (Morton and Lewis, 2007). Our further studies will focus on the mechanisms by which Rab23 expression contributes to the dysregulation of hedgehog signaling and PDAC tumorigenesis.

In conclusion, we demonstrated that Rab23 plays critical roles in PDAC invasion and metastasis, thus providing a novel potential target for the treatment of advanced PDAC.

\section{Conflicts of interest}

The authors declare no conflict of interests.

\section{ACKNOWLEDGMENTS}

Research supported by the Zhejiang Provincial Natural Science Foundation of China (\#Y2110335).

\section{REFERENCES}

Abbas S (2013). Molecular biology of adenocarcinoma of the pancreatic duct, current state and future therapeutic avenues. Surg. Oncol. 22: 69-76.

Beachy PA, Karhadkar SS and Berman DM (2004). Tissue repair and stem cell renewal in carcinogenesis. Nature 432: 324-331.

Caswell PT, Spence HJ, Parsons M, White DP, et al. (2007). Rab25 associates with alpha5beta1 integrin to promote invasive migration in 3D microenvironments. Dev. Cell 13: 496-510.

Chakraborty S, Baine MJ, Sasson AR and Batra SK (2011). Current status of molecular markers for early detection of sporadic pancreatic cancer. Biochim. Biophys. Acta 1815: 44-64.

Cheng KW, Lahad JP, Kuo WL, Lapuk A, et al. (2004). The RAB25 small GTPase determines aggressiveness of ovarian and breast cancers. Nat. Med. 10: 1251-1256.

Chi S, Xie G, Liu H, Chen K, et al. (2012). Rab23 negatively regulates Gli1 transcriptional factor in a $\mathrm{Su}(\mathrm{Fu})$-dependent manner. Cell Signal. 24: 1222-1228.

Dey P, Rachagani S, Chakraborty S, Singh PK, et al. (2012). Overexpression of ecdysoneless in pancreatic cancer and its role in oncogenesis by regulating glycolysis. Clin. Cancer Res. 18: 6188-6198.

Eggenschwiler JT, Espinoza E and Anderson KV (2001). Rab23 is an essential negative regulator of the mouse Sonic hedgehog signalling pathway. Nature 412: 194-198.

Eggenschwiler JT, Bulgakov OV, Qin J, Li T, et al. (2006). Mouse Rab23 regulates hedgehog signaling from smoothened to Gli proteins. Dev. Biol. 290: 1-12.

Guo A, Wang T, Ng EL, Aulia S, et al. (2006). Open brain gene product Rab23: expression pattern in the adult mouse brain and functional characterization. J. Neurosci. Res. 83: 1118-27.

Hou Q, Wu YH, Grabsch H, Zhu Y, et al. (2008). Integrative genomics identifies RAB23 as an invasion mediator gene in diffuse-type gastric cancer. Cancer Res. 68: 4623-4630.

Huang S, Yang L, An Y, Ma X, et al. (2011). Expression of hedgehog signaling molecules in lung cancer. Acta Histochem. 113: 564-569.

Jemal A, Siegel R, Ward E, Murray T, et al. (2006). Cancer statistics, 2006. CA Cancer J. Clin. 56: 106-130.

Jones S, Zhang X, Parsons DW, Lin JC, et al. (2008). Core signaling pathways in human pancreatic cancers revealed by global genomic analyses. Science 321: 1801-1806.

Koorstra JB, Hustinx SR, Offerhaus GJ and Maitra A (2008). Pancreatic carcinogenesis. Pancreatology 8: 110-125.

Liu Y, Tao X, Jia L, Cheng KW, et al. (2012). Knockdown of RAB25 promotes autophagy and inhibits cell growth in ovarian cancer cells. Mol. Med. Rep.6: 1006-1012. 
Liu YJ, Wang Q, Li W, Huang XH, et al. (2007). Rab23 is a potential biological target for treating hepatocellular carcinoma. World J. Gastroenterol. 13: 1010-1017.

Marcos I, Borrego S and Antinolo G (2003). Molecular cloning and characterization of human RAB23, a member of the group of Rab GTPases. Int. J. Mol. Med. 12: 983-987.

Michaud DS (2004). Epidemiology of pancreatic cancer. Minerva Chir. 59: 99-111.

Moore MJ, Goldstein D, Hamm J, Figer A, et al. (2007). Erlotinib plus gemcitabine compared with gemcitabine alone in patients with advanced pancreatic cancer: a phase III trial of the National Cancer Institute of Canada Clinical Trials Group. J. Clin. Oncol. 25: 1960-1966.

Morifuji Y, Onishi H, Iwasaki H, Imaizumi A, et al. (2014). Reoxygenation from chronic hypoxia promotes metastatic processes in pancreatic cancer through the Hedgehog signaling. Cancer Sci. 105: 324-333.

Morton JP and Lewis BC (2007). Shh signaling and pancreatic cancer: implications for therapy? Cell Cycle 6: 1553-7.

Roy I, Zimmerman NP, Mackinnon AC, Tsai S, et al. (2014). CXCL12 chemokine expression suppresses human pancreatic cancer growth and metastasis. PLoS One 9: e90400.

Silverman DT, Dunn JA, Hoover RN, Schiffman M, et al. (1994). Cigarette smoking and pancreas cancer: a case-control study based on direct interviews. J. Natl. Cancer Inst. 86: 1510-1516.

Smith AC, Heo WD, Braun V, Jiang X, et al. (2007). A network of Rab GTPases controls phagosome maturation and is modulated by Salmonella enterica serovar Typhimurium. J. Cell Biol. 176: 263-268.

Yang L, Clinton JM, Blackburn ML, Zhang Q, et al. (2008). Rab23 regulates differentiation of ATDC5 chondroprogenitor cells. J. Biol. Chem. 283: 10649-10657. 